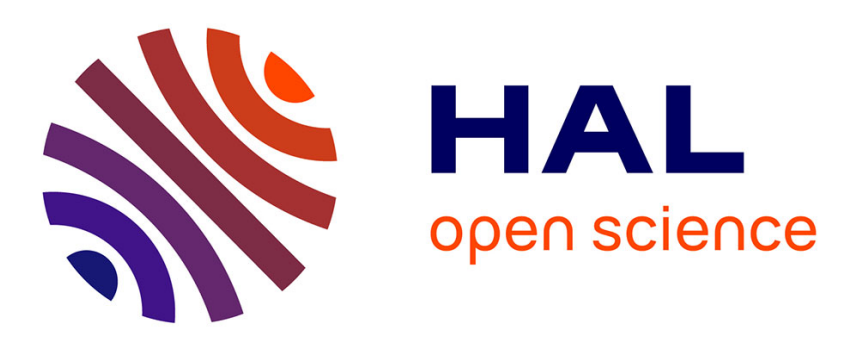

\title{
Using singular values to build a subgrid-scale model for large eddy simulations
}

Franck Nicoud, Hubert Baya Toda, Olivier Cabrit, Sanjeeb Bose, Jungil Lee

\section{To cite this version:}

Franck Nicoud, Hubert Baya Toda, Olivier Cabrit, Sanjeeb Bose, Jungil Lee. Using singular values to build a subgrid-scale model for large eddy simulations. Physics of Fluids, 2011, 23 (8), pp.085106. 10.1063/1.3623274. hal-00802472

\section{HAL Id: hal-00802472}

\section{https://hal.science/hal-00802472}

Submitted on 19 Mar 2013

HAL is a multi-disciplinary open access archive for the deposit and dissemination of scientific research documents, whether they are published or not. The documents may come from teaching and research institutions in France or abroad, or from public or private research centers.
L'archive ouverte pluridisciplinaire HAL, est destinée au dépôt et à la diffusion de documents scientifiques de niveau recherche, publiés ou non, émanant des établissements d'enseignement et de recherche français ou étrangers, des laboratoires publics ou privés. 


\title{
Using singular values to build a subgrid-scale model for Large Eddy Simulations
}

\author{
Franck Nicoud* \\ CNRS I3M 5149, University Montpellier 2. \\ Hubert Baya Toda \\ IFP Energies Nouvelles \\ Olivier Cabrit \\ CERFACS, Toulouse, France \\ Sanjeeb Bose \\ Center for Turbulence Research, Stanford, California \\ Jungil Lee \\ Seoul National University, Korea \\ (Dated: July 11, 2011)
}




\begin{abstract}
An eddy-viscosity based, subgrid-scale model for Large Eddy Simulations is derived from the analysis of the singular values of the resolved velocity gradient tensor. The proposed $\sigma$-model has by construction the property to automatically vanish as soon as the resolved field is either two-dimensional or two-component, including the pure shear and solid rotation cases. In addition, the model generates no subgrid-scale viscosity when the resolved scales are in pure axisymmetric or isotropic contraction/expansion. At last, it is shown analytically that it has the appropriate cubic behavior in the vicinity of solid boundaries without requiring any ad-hoc treatment. Results for two classical test cases (decaying isotropic turbulence and periodic channel flow) obtained from three different solvers with a variety of numerics (finite elements, finite differences or spectral methods) are presented to illustrate the potential of this model. The results obtained with the proposed model are systematically equivalent or slightly better than the results from the Dynamic Smagorinsky model. Still, the $\sigma$-model has a low computational cost, is easy to implement and does not require any homogeneous direction in space or time. It is thus anticipated that it has a high potential for the computation of non-homogeneous, wall-bounded flows.
\end{abstract}

PACS numbers: 47.10.ad, 47.27.ep, 47.27.em, 47.27.N-

\footnotetext{
* Corresponding author. Electronic address: franck.nicoud@univ-montp2.fr
} 


\section{INTRODUCTION}

When dealing with Large Eddy Simulations (LES), the eddy-viscosity assumption is by far the most used because it reduces the modeling effort considerably. In this view, the subgrid-scale (SGS) tensor is written as (the implicit summation rule for repeated indices is used throughout this paper):

$$
\tau_{i j}^{\mathrm{SGS}}-\frac{1}{3} \tau_{k k}^{\mathrm{SGS}} \delta_{i j}=2 \bar{\rho} \nu_{\mathrm{SGS}}\left(\widetilde{S}_{i j}-\frac{1}{3} \widetilde{S}_{k k} \delta_{i j}\right),
$$

where $\widetilde{S}_{i j}=\frac{1}{2}\left(\widetilde{g}_{i j}+\widetilde{g}_{j i}\right)$ and $\widetilde{g}_{i j}=\partial \widetilde{u}_{i} / \partial x_{j}$ are respectively the strain and velocity gradient tensors of the resolved scales. Note that the low pass filter used to define the resolved scales from the total field, denoted by $\bar{f}$ and used to define the mass weighted filter $\tilde{f}=\overline{\rho f} / \bar{\rho}$, will be omitted throughout this paper for simplicity. Equation 1 then reduces to:

$$
\tau_{i j}^{\mathrm{SGS}}-\frac{1}{3} \tau_{k k}^{\mathrm{SGS}} \delta_{i j}=2 \rho \nu_{\mathrm{SGS}}\left(S_{i j}-\frac{1}{3} S_{k k} \delta_{i j}\right) .
$$

From a simple dimensional analysis, it is natural to model the subgrid-scale viscosity as

$$
\nu_{\mathrm{SGS}}=\left(C_{m} \Delta\right)^{2} \mathcal{D}_{m}(\mathbf{u})
$$

where $C_{m}$ is the model constant, $\Delta$ is the subgrid characteristic length scale (in practice the size of the mesh), and $\mathcal{D}_{m}$ is a differential operator associated with the model, homogeneous to a frequency and acting on the resolved velocity field $\mathbf{u}=\left(u_{i}\right)$. The most classical operator is by far the strain rate; this leads to the Smagorinsky model [1] for which $\mathcal{D}_{m}=\mathcal{D}_{s}=$ $\sqrt{2 S_{i j} S_{i j}}$ and $C_{m}=C_{s} \approx 0.18$. This operator is known for not vanishing in near-wall regions. In the past, this major drawback motivated the use of damping functions [2], the development of the dynamic procedure [3] and other improvements such as the shearimproved Smagorinsky model [4] where the magnitude of the mean shear is assessed and removed from the local shear. It is actually possible to build invariants which do not have this drawback. Examples of such operators are used in the WALE (Wall Adapting Local Eddy viscosity) [5] and Vreman's models [6]. For these formulations, the differential operators read respectively:

$$
\mathcal{D}_{m}=\mathcal{D}_{w}=\frac{\left(\mathcal{S}_{i j}^{d} \mathcal{S}_{i j}^{d}\right)^{3 / 2}}{\left(S_{i j} S_{i j}\right)^{5 / 2}+\left(\mathcal{S}_{i j}^{d} \mathcal{S}_{i j}^{d}\right)^{5 / 4}}
$$

and

$$
\mathcal{D}_{m}=\mathcal{D}_{v}=\sqrt{\frac{G_{11} G_{22}-G_{12}^{2}+G_{11} G_{33}-G_{13}^{2}+G_{22} G_{33}-G_{23}^{2}}{g_{i j} g_{i j}}},
$$


where $G_{i j}=g_{k i} g_{k j}$ and $\mathcal{S}_{i j}^{d}$ is the traceless symmetric part of the square of the velocity gradient tensor:

$$
\mathcal{S}_{i j}^{d}=\frac{1}{2}\left(g_{i j}^{2}+g_{j i}^{2}\right)-\frac{1}{3} g_{k k}^{2} \delta_{i j}, \quad \text { with } \quad g_{i j}^{2}=g_{i k} g_{k j}
$$

Note that $\mathbf{g}$ is not symmetric so that $\mathbf{G}$ (of component $g_{k i} g_{k j}$ ) and $\mathbf{g}^{2}$ (of component $g_{i k} g_{k j}$ ) are two distinct tensors. The interesting common property shared by these two operators is that they generate zero SGS viscosity in the case of a pure shear. However, they both do not vanish in the particular case of a solid rotation (see discussion in section IID). The situation is the opposite for the Smagorinsky model which vanishes for pure rotation but not for pure shear. Thus, none of these models is satisfying since one could expect that a proper model generates zero SGS viscosity for both the pure shear and the solid rotation cases.

Independently on the properties of the underlying differential operator, all the models based on the eddy-viscosity assumption, Eq. (3), share the drawback that the model constant $C_{m}$ must be adapted to the mesh refinement so that the proper amount of energy is drained from the resolved scales. This issue is well addressed by the introduction of the dynamic procedure [3] that can automatically adapt the model constant. Besides, because the existing static eddy-viscosity based models miss some desirable properties, many treatments (connected or not to the dynamic procedure) have been proposed over the years in order to improve their performances : one can cite among many others Porté-Agel et al. [7] who proposed a double filtering procedure for removing the scale-invariance assumption usually made for computing the model constant from the dynamic procedure; Hughes et al. [8] who proposed the Variational Multi-Scale (VMS) methodology where only the smallest resolved scales are directly affected by the SGS viscosity; Jeanmart and Winckelmans [9] who proposed a regularized version of the VMS approach for use in the physical space and Bricteux et al. [10] who subsequently used the WALE operator in order to obtain a Regularized Variational Multiscale model with the proper near-wall behavior (RVM-WALE model); Shi et al. [11] who added a constraint on the modeled SGS energy flux to better represent the overall dissipation; Lodato et al. [12] who developed a scale similarity [13] version of the WALE model, using ideas introduced originally to improve the Smagorisnky model [14].

Following the framework of Lilly [15], the model constant from the dynamic procedure is 
computed resorting to a least squares approach as :

$$
\left(C_{m} \Delta\right)^{2}=-\frac{L_{i j} M_{i j}}{2 M_{i j} M_{i j}}
$$

where $L_{i j}=\widehat{u_{i} u_{j}}-\widehat{u_{i}} \widehat{u_{j}}$ is the (modified) Leonard term based on the grid-based filter (which is omitted for clarity, $u_{i}=\widetilde{u}_{i}$ ) and test filter $\widehat{\vartheta}$ In addition, $M_{i j}$ is directly related to the differential operator of the underlying eddy-viscosity model and reads:

$$
M_{i j}=\frac{\widehat{\Delta}^{2}}{\Delta^{2}} \widehat{\mathcal{D}_{m}} \widehat{S_{i j}}-\widehat{\mathcal{D}_{m} S_{i j}}
$$

where $\widehat{\Delta}$ stands for the test filter width. Unfortunately, the original dynamic procedure most often requires some averaging in order to reduce the constant variability over space and time. Several improved versions of the dynamic Smagorinsky model were proposed in order to make it more robust and suitable for complex configurations where no homogeneous directions are present $[16,17]$. Still, a common practice when dealing with complex geometries is to apply the least mean square formula over a small volume surrounding the current grid point and to clip the remaining negative values of the dynamically computed constant. This means replacing Eq. (6) by

$$
\left(C_{m} \Delta\right)^{2}=\max \left[-\frac{\left\langle L_{i j} M_{i j}\right\rangle_{\mathrm{loc}}}{2\left\langle M_{i j} M_{i j}\right\rangle_{\mathrm{loc}}}, 0\right]
$$

where $\langle\cdot\rangle_{\text {loc }}$ stands for an integral taken over a small volume (typically a few grid cells) surrounding the current grid point. Note that the model constant then depends on both space and time.

The main motivation of the local dynamic procedure was to adapt the constant to compensate the non-vanishing behavior of the Smagorinsky model in near-wall regions. Recently, Ghorbaniasl \& Lacor [18] proposed to extend the dynamic procedure to the WALE model. However, Baya Toda et al. [19] reported that the combination of the classical dynamic procedure with any SGS model that has the proper near-wall cubic behavior leads to a paradox: the underlying differential operator rapidly goes to zero near solid boundaries, which favors unstable computations. For the sake of robustness while keeping an adaptation of the model coefficient to the grid resolution and numerical errors, two concepts of global dynamic procedure emerged from the properties of the Vreman's model. The first one is based on the global equilibrium hypothesis [20] and was proposed by Park et al. [21] and later improved 
by You \& Moin [22]. The second one, based on the Germano identity, was also proposed in [21] and recently proved to be better suited for transient flows [23]. This global dynamic procedure amounts to change Eq. (6) to:

$$
\left(C_{m} \Delta\right)^{2}=-\frac{\left\langle L_{i j} M_{i j}\right\rangle_{\mathrm{dom}}}{2\left\langle M_{i j} M_{i j}\right\rangle_{\mathrm{dom}}},
$$

where $\langle\cdot\rangle_{\text {dom }}$ stands for an averaging over the whole computational domain; the model constant is then uniform over space by construction. It has the advantage of producing mostly positive values for the dynamic constant, thus avoiding the clipping issue. The price to pay is that the differential operator $\mathcal{D}_{m}$ must behave appropriately in basic flow configurations because no compensation from the dynamic procedure can be expected (the constant of the model is uniform over space). For example, such procedure is not expected to provide good results if applied to the Smagorinsky model since the eddy-viscosity would then not vanish near solid walls. The differential operators used in the WALE and Vreman models are not very appropriate either. For example, it can be shown analytically that the latter is linear with respect to the distance to solid boundaries instead of having a cubic behavior in nearwall regions. Also, they both produce non zero eddy-viscosity in simple flow configurations such as solid rotation.

From the previous discussion, Large Eddy Simulations of complex flows would benefit from the knowledge of a static SGS model with better intrinsic properties than existing formulations. Such model could be used either directly or as a first step for subsequent improvements based on the scale similarity concept, the Variational Multi-Scale framework or a (global) dynamic procedure. The objective of this paper is to propose such a static, eddy-viscosity model with improved properties. The differential operator which is used to define this model is described in section II where analytical developments are provided in order to establish the unique properties met by the proposed static SGS model. Numerical results for decaying isotropic turbulence and a periodic channel flow are shown in section III in order to illustrate the potential of the model.

\section{A SINGULAR VALUES BASED MODEL}

It would be a difficult task to establish a definite list of the desirable properties that an improved differential operator should meet. One can however draw up a set of properties 
based on basic practical/physical considerations. Similar to the Smagorinsky, WALE and Vreman's models, the operator should be defined locally, involving only local gradients of the resolved velocity field. Such property is useful both in terms of implementation in general purpose LES solvers and in terms of physical interpretation of the results. Any nonlocal effect would most probably require the computation of two-point correlations which are not easy to compute in complex flows. Moreover, it is desirable that the differential operator generates only positive values. Although negative values can be justified from a physical point of view by referring to the backscatter phenomenon, positiveness is required in this study for stability reasons. This choice was made after the observation that the local dynamic procedure, which may lead to negative SGS viscosity, Eq. (6), suffers from stability issues in complex flow configurations where averaging over homogeneous directions is not an option. Besides, it is commonly accepted that the main objective of any (eddy-viscosity based) SGS model is to drain the proper overall amount of kinetic energy from the resolved velocity scales. To this end, a positive eddy-viscosity is most probably appropriate. In what follows, positiveness and locality will be collectively referred to as Property P0.

Similar to the WALE and Vreman's models, the differential operator should tend to zero in near-wall regions in order to mimic the turbulence damping due to the no-slip condition. It can be shown that the turbulent stress, thus the eddy-viscosity and the differential operator, should decay as the distance to the solid boundary to the third power [24] [Property $\mathbf{P 1}$. At the same time, it should vanish in the case of a flow in solid rotation, like the Smagorinsky model, and in the case of a pure shear, like the WALE and Vreman models. More generally, the improved differential operator should be zero for any two-dimensional (2D) and/or two-component (2C) flows, where no subgrid scale activity is expected to occur [Property P2]. Indeed, although two-dimensional turbulence has been evidenced experimentally and numerically [25], it is a phenomenon of fundamental interest that "might $[\ldots]$ be viewed as just a toy model" [26]. Given that two- and three-dimensional turbulence are fundamentally different because of the absence of the vortex-stretching term in the former, it seems appropriate to make sure that any SGS model for three-dimensional turbulence switches off in the two-dimensional case. The alternative would be to switch to a SGS model appropriate for two-dimensional turbulence. Still, given the very little probability that a three-dimensional computation of a two-dimensional turbulent flow remains two-dimensional without any external action to maintain its two-dimensionality, this choice 


\begin{tabular}{ll}
\hline \hline P0 & a positive quantity which involves only locally defined velocity gradients \\
\hline P1 & cubic behavior near solid boundaries \\
\hline P2 & zero for any two-component or two-dimensional flows \\
\hline P3 & zero for axisymmetric or isotropic expansion/contraction \\
\hline
\end{tabular}

TABLE I. Desirable properties for an improved SGS viscosity model. In the case of a static model, these properties should be met by the differential operator $\left(\mathcal{D}_{m}\right)$ the model is based on.

is not made in this paper. Another way to justify property $\mathbf{P} 2$ is to argue that $2 \mathrm{D}$ or $2 \mathrm{C}$ resolved scales are not compatible with a subgrid-scale activity. Indeed, since the smallest resolved scales interact with subgrid scales which are presumably random-like and $3 \mathrm{D} / 3 \mathrm{C}$, they cannot remain $2 \mathrm{D}$ or $2 \mathrm{C}$ in the long run. The same reasoning leads to the conclusion that the SGS viscosity should be zero in the case where the resolved scales are either in pure axisymmetric or isotropic expansion (or contraction) [Property P3]. The former case corresponds to the situation of a laminar round jet impinging on a solid plate for which turbulent effect should indeed not be present. The latter is representative of the velocity field near an acoustic monopole or a spherical premixed flame, which again are not phenomena of turbulent nature. The desirable properties are recalled in Table I. Since they do not come from any mathematical theory of turbulence or fluid mechanics, we do not claim that they constitute a set of necessary and sufficient conditions that any SGS model should meet. Still, they seem desirable from a physical/numerical point of view as discussed above.

\section{A. Meeting Properties P2-P3}

Analyzing the spectral content of the velocity gradient tensor proves to be a proper framework to investigate how these properties can be met by a single differential operator. For example, the fact that one of the eigenvalues of $\mathbf{g}$ is zero would indicate that the flow is locally either $2 \mathrm{D}$ or $2 \mathrm{C}$. Note, however, that the eigenvalues of $\mathbf{g}$ can be complex-valued in number of flow configurations (in the case of a solid rotation, for example). Using these quantities directly to build the differential operator $\mathcal{D}_{m}$ would thus not be very convenient. One way to avoid this difficulty is to consider the strain rate tensor instead of $\mathbf{g}$. In this 
case, the three eigenvalues are real-valued, although their sign is not known a priori. This route was explored recently $[27,28]$. In the present study, one relies on the singular values of $\mathbf{g}$ to build an improved differential operator for the SGS eddy viscosity. Specifically, let us introduce $\sigma_{1} \geq \sigma_{2} \geq \sigma_{3} \geq 0$, the three singular values of $\mathbf{g}=\left(g_{i j}\right)$. As discussed in the appendix, these quantities can be computed at moderate computational cost by different means. By definition, these values are always positive and equal the square root of the eigenvalues of the matrix $\mathbf{G}=\mathbf{g}^{t} \mathbf{g}$, where the superscript $t$ denotes matrix transposition (these eigenvalues are always positive because $\mathbf{G}$ is symmetric semi-definite positive). The smallest singular value, $\sigma_{3}$, is null if and only if one row or column of $\mathbf{g}$ is zero up to a rotation of the coordinate system. In other words, $\sigma_{3}=0$ is a marker for two-dimensional and/or two-component flows, and any operator proportional to this singular value would meet property P2. Similarly, the knowledge of the singular values of $\mathbf{g}$ helps to detect the case where the resolved velocity field is in axisymmetric contraction or expansion. Indeed, an appropriate rotation of the coordinate system then makes the velocity gradient tensor diagonal:

$$
\mathbf{g}=\operatorname{diag}(\beta,-\alpha,-\alpha)
$$

where $\alpha$ is positive for a contraction and negative for an expansion. Depending on the relative values of the parameters $\alpha$ and $\beta$, the singular values of $\mathbf{g}$ read either $\sigma_{1}=|\beta|>$ $\sigma_{2}=\sigma_{3}=|\alpha|$ or $\sigma_{3}=|\beta|<\sigma_{1}=\sigma_{2}=|\alpha|$. In other words, the marker for such flow situations is either $\sigma_{2}=\sigma_{3}$ or $\sigma_{1}=\sigma_{2}$. Thus, any differential operator proportional to $\left(\sigma_{1}-\sigma_{2}\right)\left(\sigma_{2}-\sigma_{3}\right)$ would be zero as soon as the resolved velocity field is in axisymmetric contraction/expansion. The same operator would also be zero for any isotropic configuration since this situation corresponds to $\sigma_{1}=\sigma_{2}=\sigma_{3}$. At the end, such operator would meet property P3. Note that the divergence-free assumption was not made to obtain the above results ( $\beta$ not necessarily equal to $2 \alpha$ ).

\section{B. Near wall behavior}

From the above analysis, a differential operator proportional to $\sigma_{3}\left(\sigma_{1}-\sigma_{2}\right)\left(\sigma_{2}-\sigma_{3}\right)$ would meet both properties $\mathbf{P 2}$ and P3. It is now time to investigate whether property $\mathbf{P} \mathbf{1}$ is also met. This requires analyzing the asymptotic behavior of the singular values in the vicinity of a solid boundary. Without loss of generality, one may decide that this boundary is located 
within the $\left(x_{1}, x_{3}\right)=(x, z)$ plane; the normal direction to this boundary then coincides with the $x_{2}=y$ direction. Using Taylor expansions of the resolved velocity components (recall $u_{i}$ must be understood as $\left.\widetilde{u}_{i}\right)$ :

$$
\begin{aligned}
& u_{1}=a y+O\left(y^{2}\right), \\
& u_{2}=b y^{2}+O\left(y^{3}\right), \\
& u_{3}=c y+O\left(y^{2}\right)
\end{aligned}
$$

leads to the following expression for the velocity gradient tensor:

$$
\mathbf{g}=\left[\begin{array}{ccc}
a_{x} y+O\left(y^{2}\right) & a+O(y) & a_{z} y+O\left(y^{2}\right) \\
b_{x} y^{2}+O\left(y^{3}\right) & 2 b y+O\left(y^{2}\right) & b_{z} y^{2}+O\left(y^{3}\right) \\
c_{x} y+O\left(y^{2}\right) & c+O(y) & c_{z} y+O\left(y^{2}\right)
\end{array}\right]
$$

In these expressions, $O\left(y^{p}\right)$ denotes a term of order $p$ which behaves like $y^{p}$ when the distance to the solid boundary vanishes, $y \longrightarrow 0$. Moreover, subscripts denote partial derivatives (e.g.: $\left.a_{x}=\partial a / \partial x\right)$. For sake of simplicity, the first order term in the expansion of the wall normal velocity component has been zeroed. From the continuity equation, the coefficient of the neglected linear term equals

$$
\left.\frac{1}{\rho} \frac{\partial \rho}{\partial t}\right|_{y=0}
$$

since the no-slip condition imposes $\partial u_{1} / \partial x=\partial u_{3} / \partial z=0$ at $y=0$. This writing is thus strictly valid in the incompressible case and most probably well justified for flows bounded by walls submitted to stationary isothermal conditions and/or for compressible flows in the low subsonic regime.

By definition, the singular values of $\mathbf{g}$ are the square roots of the eigenvalues of $\mathbf{G}=\mathbf{g}^{t} \mathbf{g}$. Denoting by $\lambda_{1} \geq \lambda_{2} \geq \lambda_{3} \geq 0$ these eigenvalues, they are the roots of the characteristic polynomial of $\mathbf{G}$ :

$$
P(\lambda)=-\lambda^{3}+\mathcal{I}_{1} \lambda^{2}-\mathcal{I}_{2} \lambda+\mathcal{I}_{3}
$$

where the coefficients read:

$$
\begin{aligned}
& \mathcal{I}_{1}=\operatorname{tr}(\mathbf{G}), \\
& \mathcal{I}_{2}=\frac{1}{2}\left(\operatorname{tr}(\mathbf{G})^{2}-\operatorname{tr}\left(\mathbf{G}^{2}\right)\right), \\
& \mathcal{I}_{3}=\operatorname{det}(\mathbf{G}),
\end{aligned}
$$


with $\operatorname{tr}()$ and $\operatorname{det}()$ denoting the trace and the determinant of a tensor. A classical result of linear algebra is that the coefficients of Eq. (12) are tensorial invariants (they keep the same values in every coordinate system). In particular, they can be assessed either in the $(x, y, z)$ coordinate system where $\mathbf{G}$ reads:

$$
\mathbf{G}=\left[\begin{array}{ccc}
\left(a_{x}^{2}+c_{x}^{2}\right) y^{2}+O\left(y^{3}\right) & \left(a a_{x}+c c_{x}\right) y+O\left(y^{2}\right) & \left(a_{x} a_{z}+c_{x} c_{z}\right) y^{2}+O\left(y^{3}\right) \\
\left(a a_{x}+c c_{x}\right) y+O\left(y^{2}\right) & \left(a^{2}+c^{2}\right)+O(y) & \left(a a_{z}+c c_{z}\right) y+O\left(y^{2}\right) \\
\left(a_{x} a_{z}+c_{x} c_{z}\right) y^{2}+O\left(y^{3}\right) & \left(a a_{z}+c c_{z}\right) y+O\left(y^{2}\right) & \left(a_{z}^{2}+c_{z}^{2}\right) y^{2}+O\left(y^{3}\right)
\end{array}\right]
$$

or in the principal axis where $\mathbf{G}$ is simply:.

$$
\mathbf{G}=\left[\begin{array}{lll}
\lambda_{1} & & \\
& \lambda_{2} & \\
& & \lambda_{3}
\end{array}\right]
$$

In this latter case, the invariants are given by:

$$
\begin{aligned}
& \mathcal{I}_{1}=\lambda_{1}+\lambda_{2}+\lambda_{3}, \\
& \mathcal{I}_{2}=\lambda_{1} \lambda_{2}+\lambda_{1} \lambda_{3}+\lambda_{2} \lambda_{3}, \\
& \mathcal{I}_{3}=\lambda_{1} \lambda_{2} \lambda_{3} .
\end{aligned}
$$

Then, using Eq. (13) to calculate the same invariants from Eq. (14) allows obtaining the following estimates:

$$
\begin{aligned}
\lambda_{1}+\lambda_{2}+\lambda_{3} & =O\left(y^{0}\right), \\
\lambda_{1} \lambda_{2}+\lambda_{1} \lambda_{3}+\lambda_{2} \lambda_{3} & =O\left(y^{2}\right), \\
\lambda_{1} \lambda_{2} \lambda_{3} & =O\left(y^{6}\right) .
\end{aligned}
$$

A rapid examination of Eq. (14) may lead to the erroneous conclusion that the determinant of $\mathbf{G}$ should be of order $y^{4}$ instead of $y^{6}$ as reported in Eq. (17). Actually, it can readily be shown that the $y^{4}$ and $y^{5}$ terms in the Taylor expansion of $\operatorname{det}(\mathbf{G})$ are exactly zero. Since $\mathbf{G}=\mathbf{g}^{t} \mathbf{g}$ and $\operatorname{det}(\mathbf{G})=\operatorname{det}(\mathbf{g}) \times \operatorname{det}(\mathbf{g})$, the $6^{\text {th }}$ order behavior reported in Eq. (17) is also consistent with the estimate $\operatorname{det}(\mathbf{g})=O\left(y^{3}\right)$ which comes directly from Eq. (11). With the convention $\lambda_{1} \geq \lambda_{2} \geq \lambda_{3}$, the first equality in Eq. (17) imposes that $\lambda_{1}=O\left(y^{0}\right)$ and the second and third rows imply:

$$
\begin{aligned}
\lambda_{2}+\lambda_{3}+\lambda_{2} \lambda_{3} & =O\left(y^{2}\right), \\
\lambda_{2} \lambda_{3} & =O\left(y^{6}\right) .
\end{aligned}
$$


Injecting the second row of Eq. (18) into the first implies $\lambda_{2}+\lambda_{3}=O\left(y^{2}\right)$ which imposes $\lambda_{2}=O\left(y^{2}\right)$ since $\lambda_{2} \geq \lambda_{3}$ by convention. At last, the second row of Eq. (18) then leads to $\lambda_{3}=O\left(y^{4}\right)$. Eventually, since the singular values of $\mathbf{g}$ are the square roots of the $\lambda$ 's, one obtains the following estimates in the near wall region:

$$
\begin{gathered}
\sigma_{1}=O\left(y^{0}\right), \\
\sigma_{2}=O\left(y^{1}\right), \\
\sigma_{3}=O\left(y^{2}\right)
\end{gathered}
$$

\section{The $\sigma$-model}

Eqs. 19 indicate that the product $\sigma_{3}\left(\sigma_{1}-\sigma_{2}\right)\left(\sigma_{2}-\sigma_{3}\right)$ selected to meet properties $\mathbf{P} 2$ and $\mathbf{P} 3$ is of order $O\left(y^{3}\right)$ near solid boundaries and thus meets property $\mathbf{P} 1$. The derivation of the differential operator is finished by choosing a scaling factor so that a frequency scale is obtained. A natural choice is the use of the largest singular value $\sigma_{1}$, which is nothing but the norm of $\mathbf{g}$, and which would not change the asymptotic behavior of the ratio since of order $y^{0}$. Finally, the proposed differential operator and related SGS model read

$$
\begin{aligned}
& \mathcal{D}_{\sigma}=\frac{\sigma_{3}\left(\sigma_{1}-\sigma_{2}\right)\left(\sigma_{2}-\sigma_{3}\right)}{\sigma_{1}^{2}}, \\
& \nu_{\mathrm{SGS}}=\left(C_{\sigma} \Delta\right)^{2} \mathcal{D}_{\sigma}
\end{aligned}
$$

Since the three singular values are ordered such that $\sigma_{1} \geq \sigma_{2} \geq \sigma_{3} \geq 0$, this model is positive by construction. Besides, it involves only combinations of the locally defined velocity gradient tensor and thus meets property P0. It will be referred to as the $\sigma$-model in the remaining of this paper.

\section{Discussion}

Table II summarizes the properties of different differential operators and associated mod-

els. From Eqs. 5 and 13, one may note that the Vreman's model is proportional to $\sqrt{\mathcal{I}_{2}}$. Recalling that $\mathcal{I}_{2}$, the second invariant of $\mathbf{G}$, is quadratic in $y$ (see Eq. 17), the asymptotic behavior of the Vreman's model is linear in $y$ instead of being cubic. Thus, only the WALE and $\sigma$ models comply with property $\mathbf{P} \mathbf{1}$ (as well as any model based on the corresponding 
differential operator, e.g. the RVM-WALE model of [10]). Note however that the first order behavior of the Vreman's model is enough to make it more suitable for wall-bounded flows than the Smagorinsky model for which the eddy-viscosity does not tend to zero because $\mathcal{D}_{s}=O\left(y^{0}\right)$. Table II also shows that the $\sigma$-model meets properties P2-P3, contrary to the other formulations which all fail at some point. It also shares with the three other models the property to involve only locally defined velocity gradients and is thus easy to implement in any general purpose LES solver. For the Smagorinsky model, it is possible to obtain an asymptotic value of the $C_{s}$ constant by assuming an isotropic homogeneous turbulence at infinite Reynolds number and a grid-cutoff lying into the inertial range. Lilly [29] then found $C_{s}=\left(2 / 3 C_{K}\right)^{3 / 4} / \pi$ which leads to $C_{s} \approx 0.165$ if assuming the $C_{K} \approx 1.6$ for the Kolmogorov constant. Unfortunately, such theoretical analysis cannot be conducted for the other SGS models and the model constants are usually obtained from numerical experiments. The value reported for the model constant, $C_{\sigma} \approx 1.35$, is a rough assessment generated by equating the averaged SGS dissipation obtained by feeding the Smagorinsky model and Eq. (20) with a large sample of random velocity gradient tensors. Interestingly, this crude random procedure used to provide a first assessment of $C_{\sigma}$ leads to fair estimates of the WALE and Vreman's constants $\left[C_{w} \approx 0.57\right.$ and $C_{v} \approx 0.26$, to be compared with the values recommended by Nicoud and Ducros [5] and Vreman [6] and reported in Table II]. Besides, computations of decaying isotropic turbulence confirm this value, as discussed in section III.

\section{NUMERICAL EXPERIMENTS}

Two academic configurations were considered in order to test the capability of the proposed SGS model, namely the decaying isotropic turbulence case and the periodic channel flow. In each case, LES results are compared to either experimental or Direct Numerical Simulation (DNS) data. For the channel flow case, the $\sigma$-model was also benchmarked against the dynamic Smagorinsky model. At last, in order to make sure that the conclusions drawn in terms of SGS model potential do not depend on a specific numerical method, different solvers were used during the course of this study. The three solvers considered are the following: 
Model Smagorinsky WALE Vreman $\sigma$-model

$[1] \quad[5] \quad[6] \quad$ Present

\begin{tabular}{ccccc} 
Operator & $\sqrt{2 S_{i j} S_{i j}}$ & Eq. (4) & Eq. (5) & Eq. (20) \\
Model constant & $C_{s} \approx 0.165$ & $C_{w} \approx 0.50$ & $C_{v} \approx 0.28$ & $C_{\sigma} \approx 1.35$ \\
\hline P0 & YES & YES & YES & YES \\
\hline Asymptotic & $O\left(y^{0}\right)$ & $O\left(y^{3}\right)$ & $O(y)$ & $O\left(y^{3}\right)$ \\
P1 & NO & YES & NO & YES \\
\hline Solid rotation & 0 & $\approx 0.90$ & $\approx 0.71$ & 0 \\
Pure shear & 1 & 0 & 0 & 0 \\
P2 & NO & NO & NO & YES \\
\hline Axisymmetric & $\approx 3.46$ & $\approx 0.15$ & $\approx 1.22$ & 0 \\
Isotropic & $\approx 2.45$ & 0 & 1 & 0 \\
P3 & NO & NO & NO & YES
\end{tabular}

TABLE II. Properties of the SGS models considered. Labels Axisymmetric and Isotropic refer to axisymmetric and isotropic contraction/expansion respectively. The numerical entries in the $\mathbf{P 2}$ and $\mathbf{P 3}$ blocks are the values taken by the differential operators when all the velocity derivatives are zero except: Solid rotation: $d u_{1} / d x_{2}=-1$ and $d u_{2} / d x_{1}=1$; Pure shear: $d u_{1} / d x_{2}=1$; Axisymmetric: $d u_{1} / d x_{1}= \pm 2, d u_{2} / d x_{2}=\mp 1, d u_{3} / d x_{3}=\mp 1$; Isotropic: $d u_{1} / d x_{1}= \pm 1, d u_{2} / d x_{2}=$ $\pm 1, d u_{3} / d x_{3}= \pm 1$

- Solver A: The general purpose AVBP code developed at CERFACS and IFP Energies Nouvelles solves the compressible Navier-Stokes equations. It is based on a cell-vertex formulation and embeds a set of finite element/ finite volume schemes for unstructured meshes [30, 31]; its efficiency and accuracy have been widely demonstrated for flow configurations with [32] or without [33] chemical reaction. In the present study a centered Galerkin finite element method ( $4^{\text {th }}$ order in space) with a $3^{\text {rd }}$ order RungeKutta temporal integration is retained for the investigation of two configurations: the decaying isotropic turbulence from the Comte-Bellot \& Corrsin[34] (CBC) experiment and a periodic turbulent channel flow at low subsonic Mach number. These flows were computed with the Dynamic Smagorinsky model and the present static $\sigma$-model. 
The dynamic procedure was applied locally, without averaging over homogeneous directions. Negative values of the dynamically tuned constant were clipped to ensure stability (see Eq. 7).

- Solver B: a finite difference code dedicated to the computation of turbulent channels and developed at the Center for Turbulence Research. It is based on a kinetic energy conserving, $4^{\text {th }}$ order scheme in space as proposed by Morinishi et al. (1998)[35]. A $3^{\text {rd }}$ order Runge-Kutta scheme is used for the time integration, except for the diffusion terms in the direction normal to the wall that are integrated thanks to an CrankNicholson scheme. The divergence-free condition is met by a projection scheme. It was used to compute a periodic turbulent channel flow case with both the dynamic Smagorinsky model and the present static $\sigma$-model. Note that contrary to the implementation used in the general purpose AVBP solver, the dynamic procedure is not applied locally in this case. Instead, the Smagorinsky constant is computed as

$$
\left(C_{s} \Delta\right)^{2}=-\frac{\left\langle L_{i j} M_{i j}\right\rangle_{\text {plane }}}{2\left\langle M_{i j} M_{i j}\right\rangle_{\text {plane }}}
$$

where $\langle\cdot\rangle_{\text {plane }}$ stands for an integral taken over homogeneous planes parallel to the walls of the channel. This avoids clipping while keeping the favorable dependence of the model constant on the distance to the solid boundaries.

- Solver C: a dealiased spectral code developed at Seoul National University [23]. It is based on a $2^{\text {nd }}$ order semi-implicit scheme for time integration: diffusion terms are treated implicitly using the Crank-Nicolson method, and a $3^{\text {rd }}$ order Runge-Kutta scheme is applied to convection terms. The decaying isotropic turbulence from the Comte-Bellot \& Corrsin [34] and the Kang et al.[36] experiments were computed with a dynamic version of the $\sigma$-model. The Germano-based global dynamic procedure $[21,23]$ was used (see Eq. 8), meaning that a single-model constant was computed for the whole domain at each time step. The divergence-free initial field was generated using an appropriate re-scaling method [36].

Note that the $\sigma$-model was implemented in other solvers [37], including another general purpose LES solver developed at the CORIA lab (Rouen, France) and a pseudo-spectral solver developed at the LEGI lab (Grenoble, France). Only results from the three solvers 
Solver A

\begin{tabular}{|c|c|c|c|}
\hline Key reference & {$[30,31]$} & {$[35]$} & {$[23]$} \\
\hline $\begin{array}{l}\text { Spatial } \\
\text { scheme }\end{array}$ & $\begin{array}{l}\text { - Fourth-order Galerkin } \\
\text { - finite element/finite } \\
\text { volume }\end{array}$ & $\begin{array}{l}\text { - Fourth-order } \\
\text { - kinetic energy } \\
\text { conserving } \\
\text { - finite differences }\end{array}$ & - Dealiased spectral \\
\hline $\begin{array}{c}\text { Time } \\
\text { integration }\end{array}$ & - RK & $\begin{array}{l}\text { - RK } \\
\text { - CN for diffusion } \\
\text { (wall normal) }\end{array}$ & $\begin{array}{l}-\mathrm{RK} \\
\text { - CN for diffusion }\end{array}$ \\
\hline $\begin{array}{l}\text { Dynamic } \\
\text { procedure }\end{array}$ & $\begin{array}{l}\text { - local averaging } \\
\text { - clipping required } \\
\text { - Eq. (7) }\end{array}$ & $\begin{array}{l}\text { - plane averaging } \\
\text { - clipping optional } \\
\text { - Eq. (21) }\end{array}$ & $\begin{array}{l}\text { - global averaging } \\
\text { - no clipping } \\
\text { - Eq. (8) }\end{array}$ \\
\hline
\end{tabular}

TABLE III. Properties of the solvers A, B and C used to compute the academic cases considered. RK: Third-order Runge-Kutta. CN: Second-order Crank-Nicolson

A-C described above are shown in this paper for sake of simplicity and because the same trends were observed independently on the numerical tools. The main characteristics of the solvers used in the following are gathered in Table III.

\section{A. Isotropic decaying turbulence}

We first validate the behavior of the $\sigma$-model for the simple case of a freely decaying isotropic homogeneous turbulence. The experiment by Comte-Bellot \& Corrsin [34] on decaying turbulence behind a grid is simulated first, where the mesh size of the grid turbulence is $M=5.08 \times 10^{-2} \mathrm{~m}$ and the free-stream velocity is $U_{0}=10 \mathrm{~m} / \mathrm{s}$. The Taylor micro-scale Reynolds number is $R_{\lambda}=u_{r m s} \lambda / \nu=71.6$ at time $t U_{0} / M=42$ and decreases to 60.6 at $t U_{0} / M=171$. In a reference frame moving with the average flow velocity the 
problem can be thought of as freely decaying isotropic turbulence. We model this by considering the fluid to be inside a cube-shaped box with periodic boundary conditions and size $(11 M)^{3}$. The flow was first computed with the general purpose code AVBP (Solver A), where the static $\sigma$-model was implemented. Several values of the model constant were tested, $C_{\sigma}=1.3 ; 1.4 ; 1.5 ; 1.6 ; 1.7$, for two grid resolutions, namely $48^{3}$ and $64^{3}$. The corresponding computational mesh size-to-Kolmogorov length scale ratio $\Delta / \eta$ are 40 and 30 respectively. The computations are initialized with a synthetic turbulent field whose energy spectrum is the experimental one at reduced time $t U_{0} / M=42$. The time evolutions of the resolved kinetic energy obtained from the 10 simulations (two grid resolutions and 5 constant values) are shown in Figure 1. For the $64^{3}$ grid resolution, the best agreement with experimental data at times $t U_{0} / M=98$ and $t U_{0} / M=171$ is obtained for $C_{\sigma} \approx 1.5$ whereas a larger value, in the range $1.6-1.7$ seems more adequate for the $48^{3}$ case. Note that the dependency of the model constant on the grid resolution is a common drawback of all static SGS models (and to a less extend of dynamic models when using very coarse grids) and is not specific to the $\sigma$-model (see Cocle et al. [38] for a detailed discussion on the dependence of several model constants on $\Delta / \eta)$. Note also that the increase of the model constant with the increase of the $\Delta / \eta$ ratio is coherent with previous studies [38, 39]. Figure 2 (top plot) shows that the computed spectra obtained for $C_{\sigma}=1.5$ and the $64^{3}$ grid resolution are in fair agreement with the experimental data. The biggest differences are obtained for the smallest scales; they are most probably due the large numerical errors that characterize finite volume/finite element methods for large wave numbers. To confirm this statement, an additional computation was performed with the spectral Solver C. As shown in Figure 2 (bottom plot), the agreement with the experimental spectra is now very good even for the smallest resolved scales, although the grid resolution was even coarser $\left(32^{3}, \Delta / \eta \approx 60\right)$. Note that the global dynamic $\sigma$-model was used in this case, as another mean to establish the appropriate $C_{\sigma}$ value. More precisely, the global dynamic procedure based on the Germano identity and proposed by Park et al.[21] and Lee et al.[23] was used in order to compute the constant value (homogeneous in space) at each time step. The time evolution of the dynamically tuned constant is displayed in Figure 3. Because an appropriate re-scaling method [36] was used to generate the initial velocity field at time $t U_{0} / M=42$, there is no strong variations of the computed constant during the first instants of the simulation. Moreover, the range of variation of the constant throughout the computation is roughly 1.4-1.7, consistently with 
the value suggested by the computations performed with Solver A (fig. 1). To further support this result and make sure that the $\sigma$-model can handle demanding LES (with larger $\Delta / \eta$ ratio and Reynolds number), the isotropic decaying turbulence of Kang et al.[36] was also simulated. In this case, the mesh size of the experimental grid turbulence is $M=0.152 \mathrm{~m}$ and the free-stream velocity is $U_{0}=11.2 \mathrm{~m} / \mathrm{s}$. The Taylor micro-scale Reynolds number is $R_{\lambda}=716$ at location $x / M=20$ (or time $t U_{0} / M=20$ ) and decreases to 626 at $x / M=48$ (or $t U_{0} / M=48$ ). Solver $\mathrm{C}$ was used to solve the flow equations in a computational domain of size $(33.7 M)^{3}$ at grid resolution $128^{3}$; this corresponds to a $\Delta / \eta \approx 360$. This computation is thus substantially more challenging than the $32^{3}$ Comte-Bellot \& Corrsin case in terms of SGS modeling, with a Reynolds number and $\Delta / \eta$ ratio 10 and 6 times larger respectively. The corresponding time evolution of the dynamically tuned constant is also displayed in Figure 3; it is found to be smaller in this case, close to 1.35. Given the large values of the Reynolds number and $\Delta / \eta$ ratio, it is fair to consider this later value as close to the asymptotic [38] $C_{\sigma}$ value to be used for demanding LES $(\Delta / \eta>100)$ of fully turbulent flows; as a matter of fact, $C_{\sigma} \approx 1.35$ is also the value obtained from the random procedure used in section IID (see Table II).

Despite a larger $\Delta / \eta$ ratio, this value is smaller than the range of values obtained from the Comte-Bellot \& Corrsin case at $32^{3}$ grid resolution $(1.4-1.7)$. Contrary to what may be erroneously concluded, this behavior is not contradictory with the expected increase [38, 39] of the model constant for increasing $\Delta / \eta$. Instead, the observed decrease is most probably due to small values taken by the $L / \Delta$ ratio, where $L=k^{3 / 2} / \epsilon$ is the integral length scale based on the total turbulent kinetic energy $k$ and its dissipation $\epsilon$. Recall that the constant of the Smagorinsky model increases strongly with $L / \Delta$ when the latter is in the low range, $L / \Delta<10$ say [40,41]; the same trend is expected to hold for any eddy-viscosity based subgrid-scale model, although the critical value of $L / \Delta$ above which the model constant becomes independent on this ratio may change from one model to the other. Now, given that $L / \Delta$ is only 2.6 for the $32^{3}$ Comte-Bellot \& Corrsin case while it reaches 13.4 for the case of Kang et al. with $128^{3}$ grid resolution, it is expected that the model constant significantly varies due to $L / \Delta$ when going from the Comte-Bellot \& Corrsin to the Kang's case. In other words, the observed decrease of $C_{\sigma}$ most probably results from the $\Delta / \eta$ effect being offset by the $L / \Delta$ influence.

The computed spectra at time $t U_{0} / M=30,40$ and 48 are shown in figure 4 together 

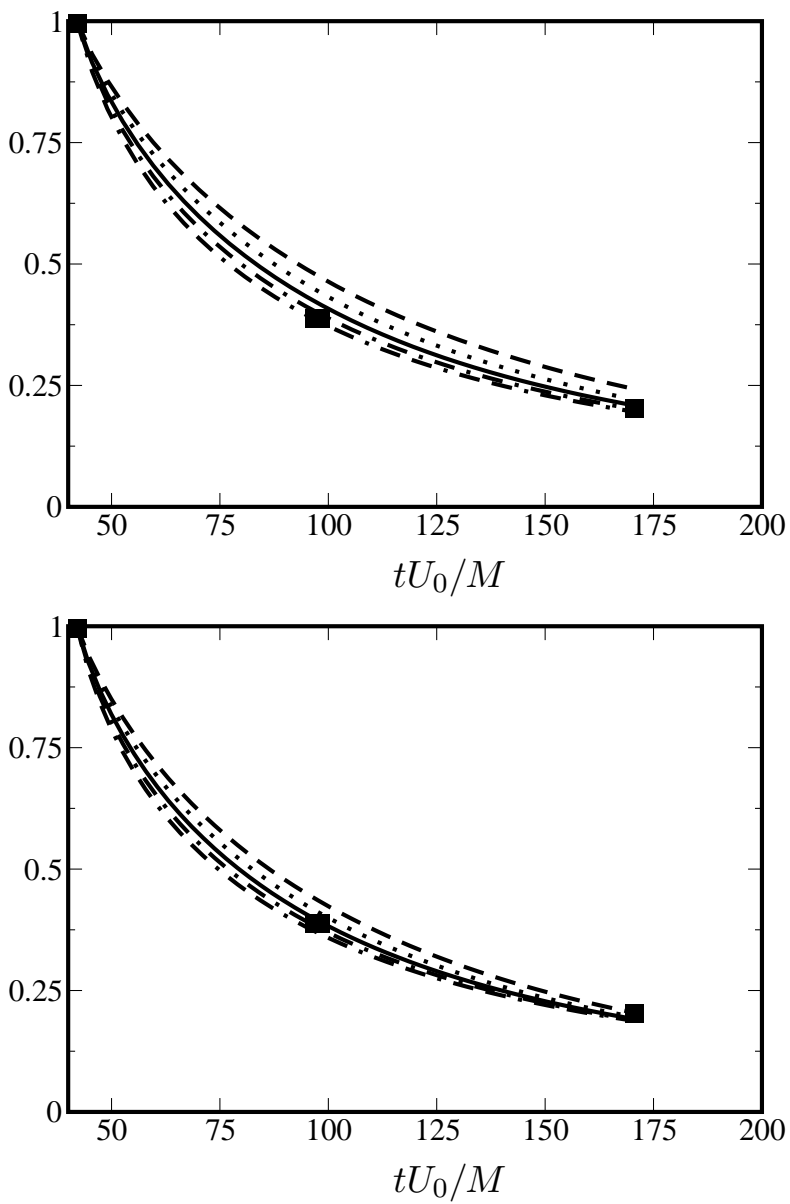

FIG. 1. Time evolutions of the scaled kinetic energy for the freely decaying isotropic turbulence corresponding to the Comte-Bellot \& Corrsin [34] experiment. All computations performed with the general purpose solver AVBP (Solver A) and the $\sigma$-model with $C_{\sigma}=1.3(---)$; $C_{\sigma}=1.4$ $(\cdots \cdots \cdots) ; C_{\sigma}=1.5(-) ; C_{\sigma}=1.6(-\cdot-) ; C_{\sigma}=1.7(--\cdot) ;$. Symbols are experimental measurements corresponding to the three-dimensionless times $t U_{0} / M=42,98$ and 171 . Top: $48^{3}$ grid resolution. Bottom: $64^{3}$ grid resolution.

with the experimental data [36]. The overall agreement is again quite good for most of the wavenumbers. The energy rise near the largest wavenumber is a well-known behavior for approaches where the same eddy-viscosity is applied to the whole range of scales and has motivated the development of spectral eddy viscosity [42] and multiscale [8, 9] models. From all the results presented in this section, it is fair to propose $1.3-1.5$ as a reasonable range of values for the $C_{\sigma}$ constant of the $\sigma$-model (Eq. 20). 

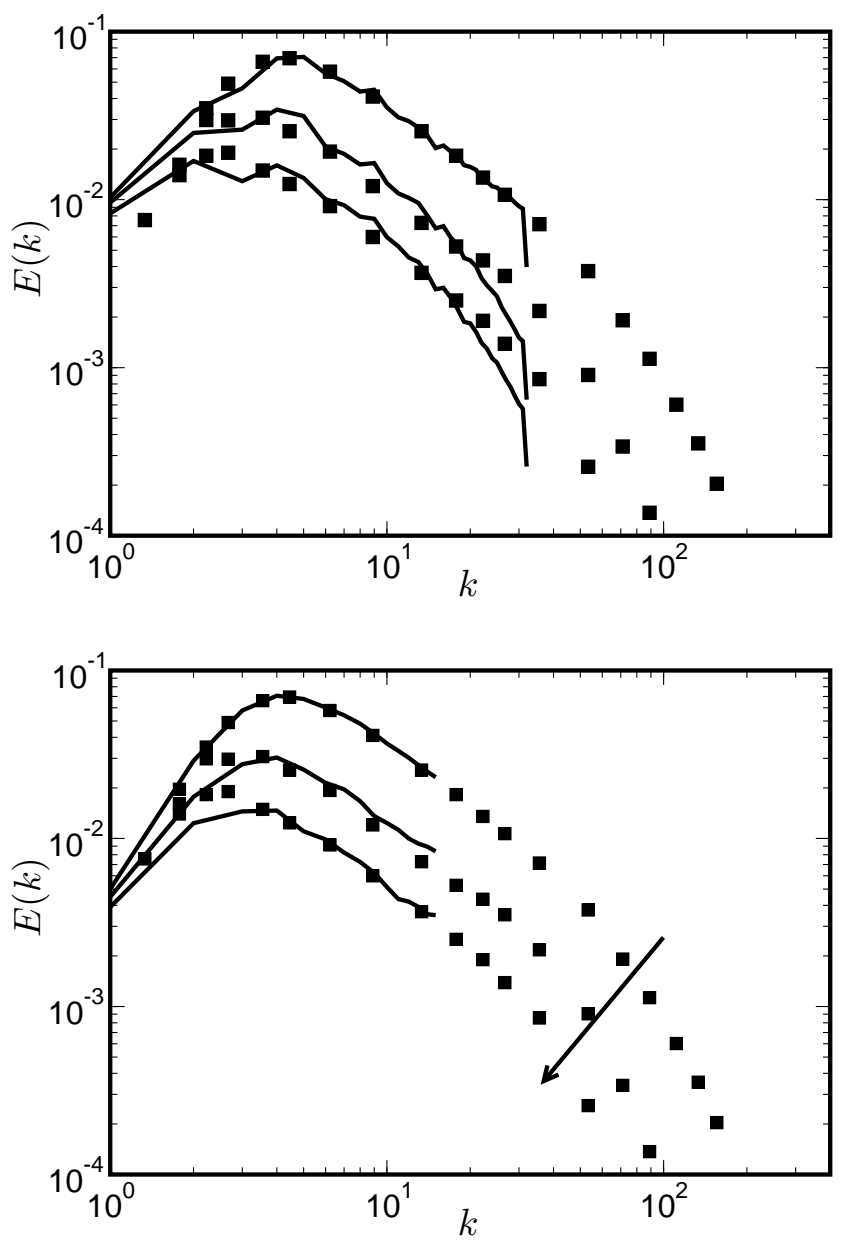

FIG. 2. Time evolutions of energy spectra for the freely decaying isotropic turbulence corresponding to the Comte-Bellot \& Corrsin [34] experiment. Symbols are experimental measurements corresponding to the three-dimensionless times $t U_{0} / M=42,98$ and 171. Top: Results from the general purpose solver AVBP (Solver A) with grid resolution $64^{3}$ and $C_{\sigma}=1.5$. Bottom: Results from a spectral method (Solver C) with grid resolution $32^{3}$ and a global dynamic procedure applied to the $\sigma$-model.

\section{B. Turbulent Channel flow}

The performance of the static $\sigma$-model for wall-bounded flows was investigated by computing LES of turbulent channel flows at friction Reynolds number $R_{\tau}=395$ and 590 . As usual, $R_{\tau}=u_{\tau} h / \nu$ with $u_{\tau}$ being the friction velocity, $h$ the channel half-height and $\nu$ the molecular kinematic viscosity. Two different solvers were used for studying the channel flow configuration, namely solvers A and B (see Table III). For sake of simplicity, only results 


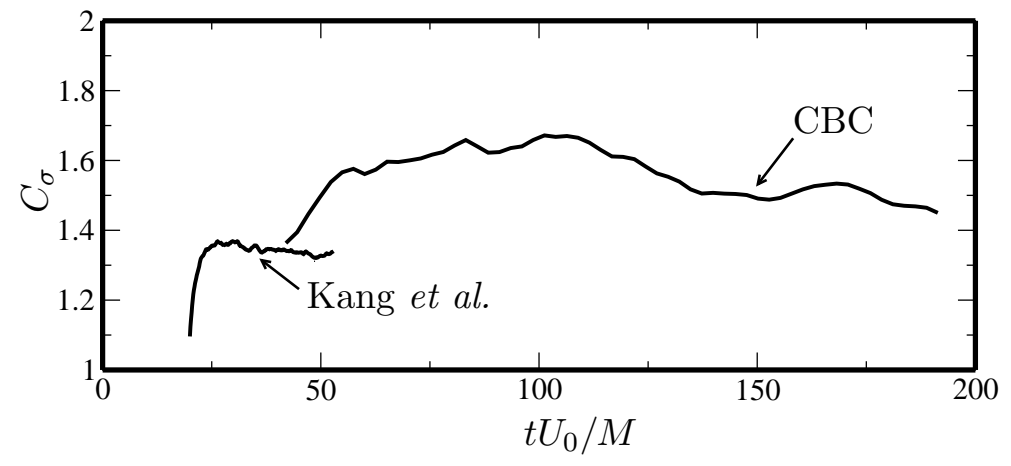

FIG. 3. Time evolution of model constant for the freely decaying isotropic turbulence corresponding to the Comte-Bellot \& Corrsin (CBC) [34] and Kang et al.[36] experiments. The computation is based on a spectral method (Solver $\mathrm{C}$ ) and a global dynamic version of the $\sigma$-model. Grid resolution is $32^{3}$ and $128^{3}$ respectively.

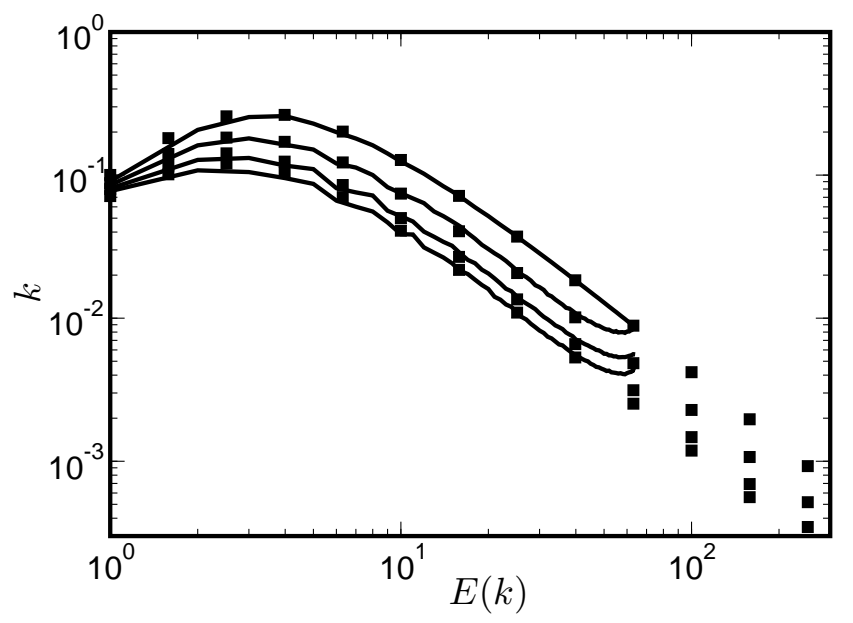

FIG. 4. Time evolutions of energy spectra for the freely decaying isotropic turbulence corresponding to the Kang et al.[36] experiment. Symbols are experimental measurements corresponding to the three-dimensionless times $t U_{0} / M=20,30,40$ and 48. Results are from the spectral Solver $\mathrm{C}$ with grid resolution $128^{3}$ and a global dynamic procedure applied to the $\sigma$-model.

from Solver $\mathrm{A}$ at $R_{\tau}=395$ and from Solver $\mathrm{B}$ at $R_{\tau}=590$ are discussed in the following. Classical values for the size of the computational domain and the grid resolution have been used, as reported in Table IV. Note however that the grid resolution is only marginal for the 590 case, especially in the spanwise direction: $\Delta z^{+} \approx 30$ while 20 wall units would be more appropriate for a fair representation of the near-wall elongated structures with a 


\begin{tabular}{ccccccccccc}
$R_{\tau}$ & Solver & $L_{x}$ & $L_{y}$ & $L_{z}$ & $N_{x}$ & $N_{y}$ & $N_{z}$ & $\Delta x^{+}$ & $\Delta y^{+}$ & $\Delta z^{+}$ \\
\hline \hline 395 & $\mathrm{~A}$ & 3.5 & 2 & 1.3 & 30 & 138 & 50 & 48 & $1-17$ & 10 \\
590 & $\mathrm{~B}$ & 6.3 & 2 & 3.1 & 64 & 64 & 64 & 58 & $0.7-50$ & 29 \\
\hline
\end{tabular}

TABLE IV. Properties of the channel flow cases. $L_{x}, L_{y}$ and $L_{z}$ stand for the size of the computational domain in the streamwise, normalwise and spanwise directions and are scaled by the channel half-height $h ; N_{x}, N_{y}$ and $N_{z}$ are the corresponding number of grid cells. Grid spacings are given in classical wall units; the mesh is stretched in the $y$-direction to increase resolution in the near wall regions. $R_{\tau}$ stands for the friction Reynolds number based on the friction velocity and channel half-height.

fourth-order scheme as in Solver B. Additional computations with twice finer spanwise resolution $\left(\Delta z^{+} \approx 15\right)$ with or without refinement in the streamwise direction were performed. The corresponding results (not shown) confirm the good behavior of the $\sigma$-model which will be illustrated in the remaining of this section. Besides, in order to properly represent the steep gradients in the viscous and buffer layers, the mesh is stretched in the wall normal direction by using either a geometric progression with common ratio close to 1.04 (Solver A) or an hyperbolic tangent law with stretching parameter close to 2.9 (Solver B). At last, the $\Delta^{2}$ term in the eddy-viscosity, Eq. (3), was computed as the $2 / 3$-power of the local cell volume. In all cases, statistics were accumulated over more than 10 diffusion times $h / u_{\tau}$ and their convergence was checked by looking at the symmetry of the profiles over the channel height. In each case, the results from the $\sigma$-model with $C_{\sigma}=1.5$ were compared with the (filtered) DNS data from Moser et al.[43]. They were also compared to the results from an implementation of the dynamic Smagorinsky model, as available in each solver (see Table III).

All the LES mean velocity profiles are in good agreement with the reference data from the available DNS, as displayed in Fig. 5. The largest difference is obtained for the $R_{\tau}=395$ case computed with solver A and the dynamic Smagorinsky model. This is most probably due to the fact that the Germano identity is applied locally in this general purpose solver (see Table III). To ensure numerical stability, it is then necessary to clip the negative values obtained for the SGS viscosity, using Eq. (7). A closer look at this computation shows that the clipping process is activated for approximately one-third of the constant computations. 
It is then not surprising that the overall quality of the results degrades, as observed in Figure 5. On the other hand, when the dynamic procedure is applied planewise, accounting for the homogeneous directions in the channel flow configuration, the clipping operation is virtually never activated. Consistently, the results from the dynamic Smagorinsky model as implemented in the dedicated solver $\mathrm{B}\left(R_{\tau}=590\right.$ case $)$ are in very good agreement with the filtered DNS data. Because the $\sigma$-model is positive and has the proper near wall behavior, it requires no clipping and the corresponding results are good in both cases. This point is confirmed by Figure 6 which displays the velocity fluctuations. Surprisingly, the situation is opposite to Figure 5, with the overall agreement being better for the $R_{\tau}=395$ case. Some kind of modeling/numerical error compensation might be the reason why the dynamic Smagorinsky implementation of solver A gives better results in terms of rms than mean velocity. Interactions between different sources of errors are expected to occur in LES but are out of the scope of this paper. On the other hand, results from the $\sigma$-model $\left(R_{\tau}=395\right)$ are just as good as for the mean velocity profile. As far as the $R_{\tau}=590$ case is concerned, discrepancies with the filtered DNS data are larger, especially for the rms velocity in the streamwise direction for which the two models considered give equivalent results. Note however that the $\sigma$-model leads to some improvement in the profiles of the spanwise and wall normal velocity fluctuations. As expected, a clear improvement was also observed when using a finer mesh in the spanwise direction $\left(\Delta z^{+} \approx 15\right.$ instead of 29 in Fig. 6). The maximum of streamwise rms velocity in then approx. 2.8 wall units, in better agreement with the DNS data (the maximum is approx. 3.1 for $\Delta z^{+}=29$ against 2.7 for the filtered DNS, see Fig. 6).

It is often accepted that the asymptotic behavior of the SGS viscosity in near wall regions is an important factor when dealing with wall resolved LES. Thus, the different computations were post-processed and the resulting behaviors plotted in Fig. 7. The theoretical behavior of the $\sigma$-model near solid boundaries $\left(\nu_{\mathrm{SGS}}=O\left(y^{3}\right)\right)$ is well retrieved numerically. Note that the amount of SGS eddy-viscosity is not negligible in front of the molecular viscosity, at least in the core region. This reflects the fact that the grid resolution is far from what is required to perform DNSs of the same flows (see Table IV). This is illustrated by the no-model calculation performed in the $R_{\tau}=590$ case (see figure 5 ) which shows measurable (although not huge) error compared to the $\sigma$-model result (the no-model velocity profile being roughly $5 \%$ smaller than the filtered DNS profile over most of the channel height). 

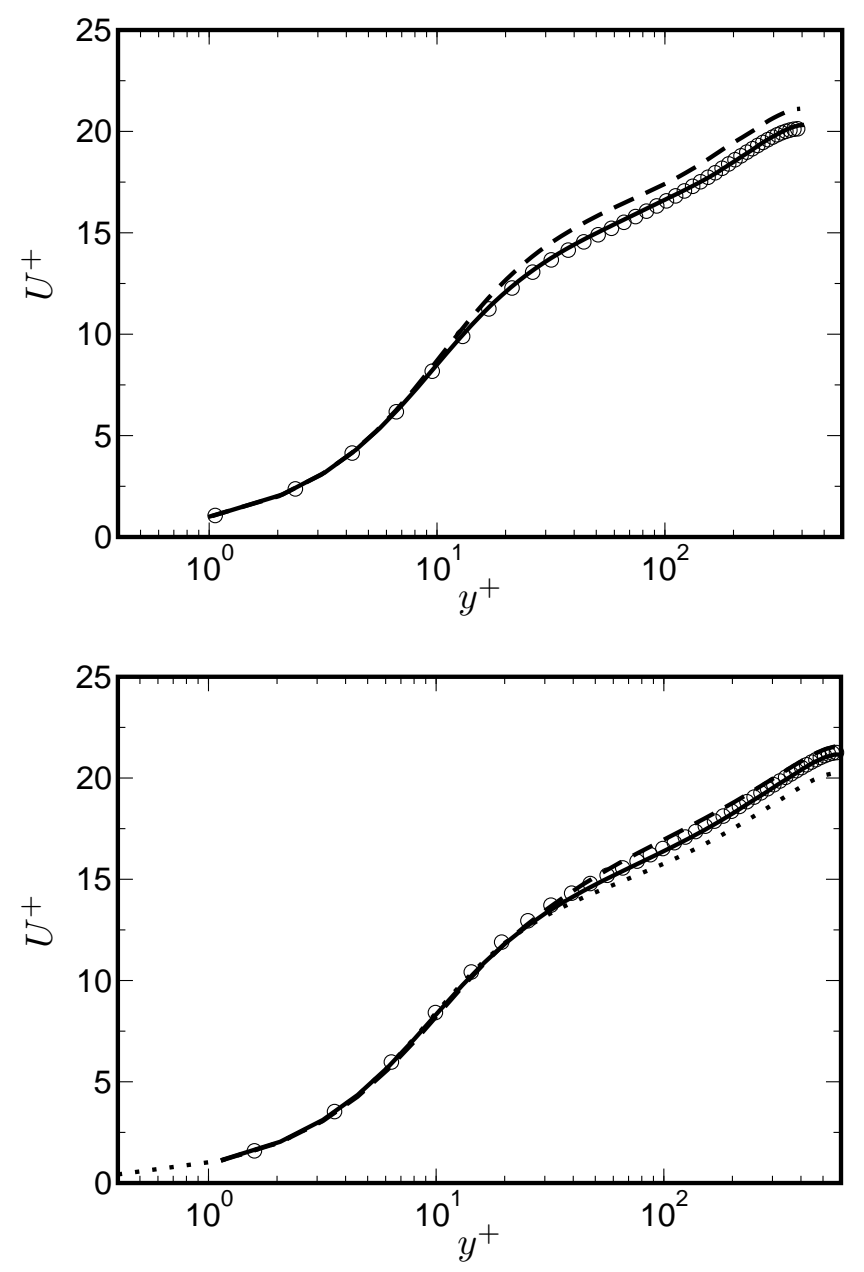

FIG. 5. Mean velocity profile from the static $\sigma$-model $(-)$ and the dynamic Smagorinsky model (--- ). Symbols correspond to the filtered DNS data [43]. Top: Results from the general purpose solver AVBP (Solver A) at $R_{\tau}=395$. Bottom: Results from the channel code (Solver B) at $R_{\tau}=590$. A no-model simulation $(\cdots \cdots \cdots)$ is also shown in this latter case.

Another indication of the effectiveness of the SGS contribution is that the no-model computation proved unstable with Solver A which is not kinetic energy conserving, contrary to Solver B. Figure 7 also illustrates that the proper asymptotic behavior is obtained with the dynamic Smagorinsky model only when the plane-wise procedure (Eq. 21) is applied, as for the case $R_{\tau}=590$ and Solver B. Recall that this procedure can be used only for simple cases with homogeneous directions. Conversely, the asymptotic behavior is built in the $\sigma$-model's differential operator itself and no specific dynamic procedure/homogeneous directions is required. 

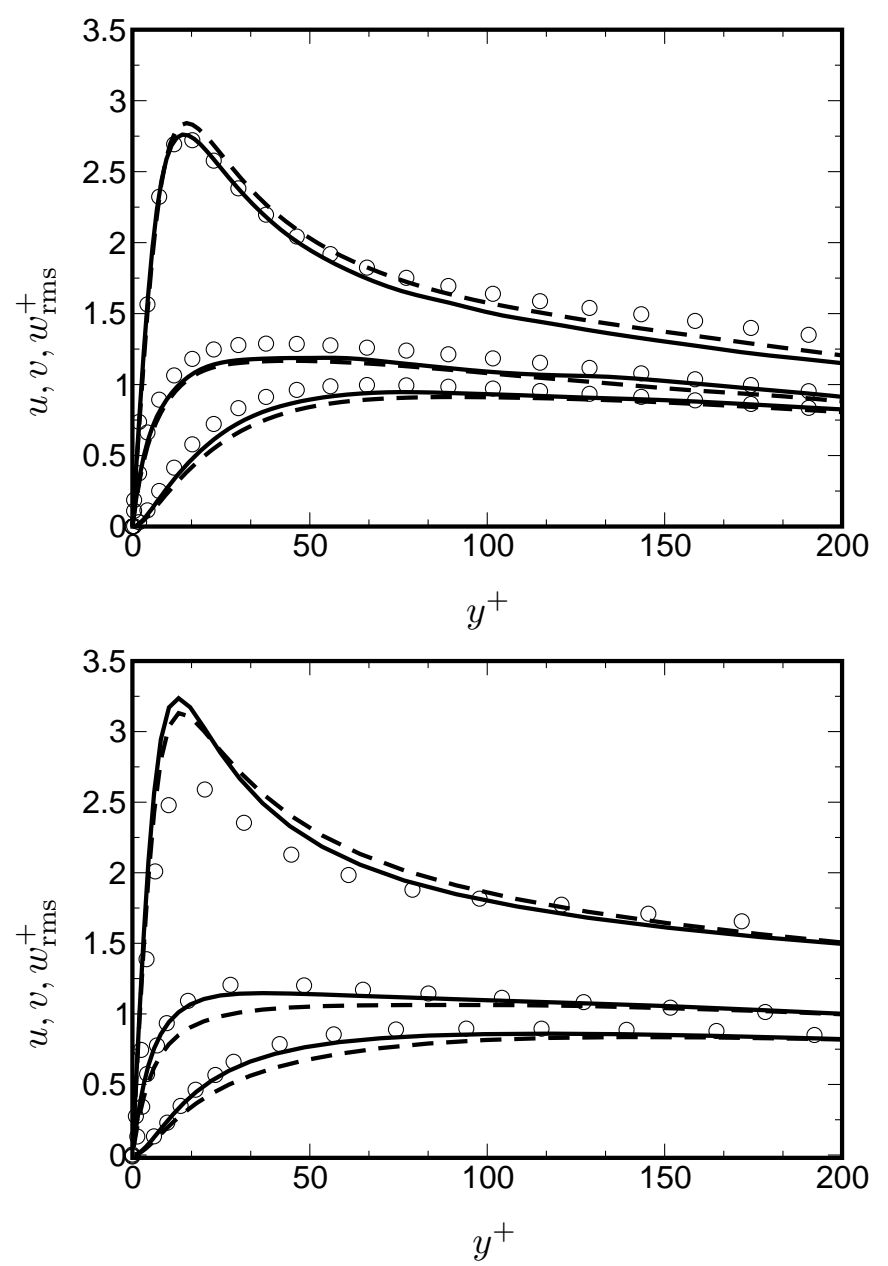

FIG. 6. Velocity fluctuations (rms) from the static $\sigma$-model (—) and the dynamic Smagorinsky model (--- ). Symbols correspond to the filtered DNS data [43]. Top: Results from the general purpose solver AVBP (Solver A) at $R_{\tau}=395$. Bottom: Results from the channel code (Solver B) at $R_{\tau}=590$.

\section{CONCLUSIONS}

A differential operator based on the singular values of the velocity gradient tensor is proposed as a basis for an improved SGS eddy-viscosity model. It is shown that the proposed static $\sigma$-model generates zero eddy-viscosity for any two-dimensional or two-component flows, as well as for axisymmetric and isotropic compressions/expansions. It also has the proper cubic behavior in near-wall regions. Implemented in three LES solvers with different numerics, the model gave promising results for two academic configurations. Owing to its unique properties, ease of implementation and low computational cost, it is anticipated that 

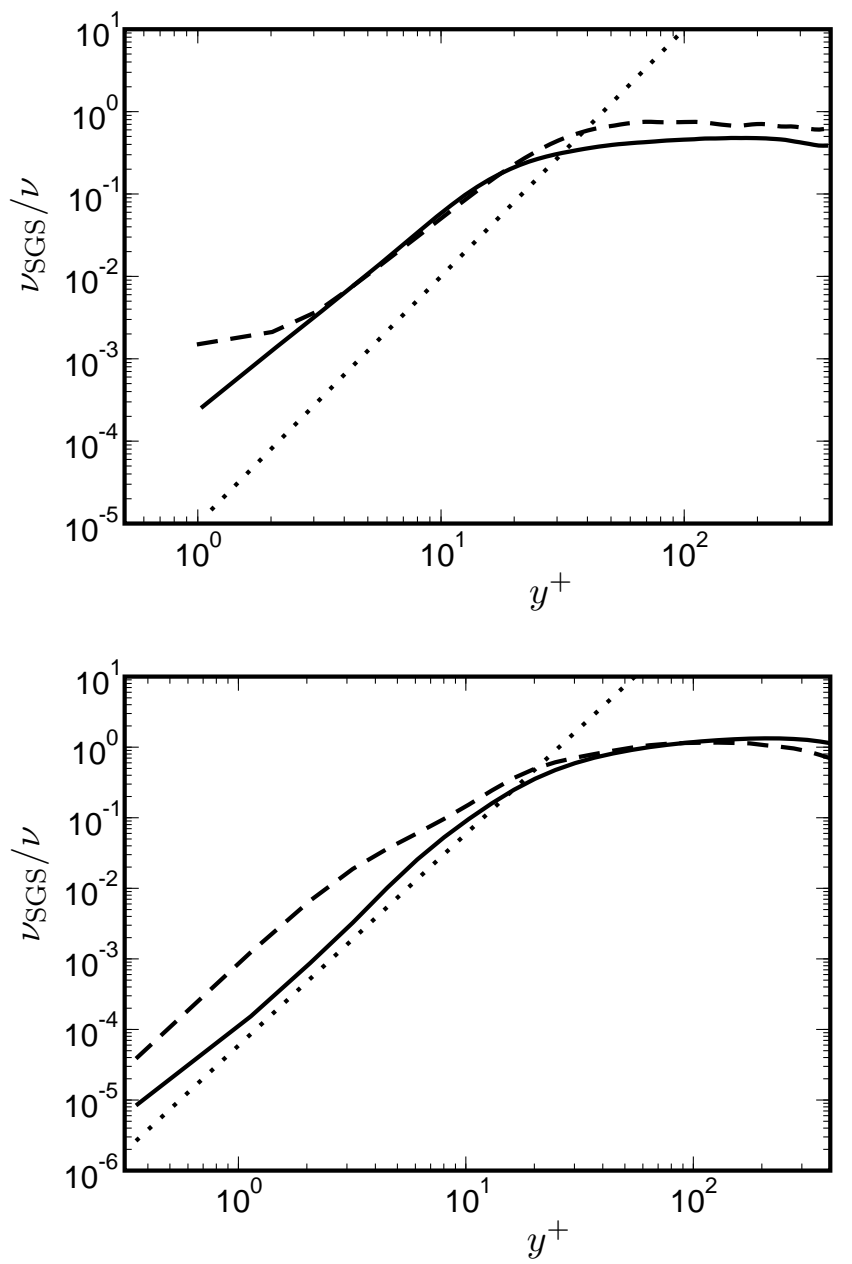

FIG. 7. Scaled SGS eddy-viscosity from the static $\sigma$-model (- ) and the dynamic Smagorinsky model (--- ). The dotted lines correspond to the proper $y^{3}$ asymptotic behavior. Top: Results from general purpose solver AVBP (Solver A) at $R_{\tau}=395$. Bottom: Results from the channel code (Solver B) at $R_{\tau}=590$.

the $\sigma$-model could be useful in the current effort to make LES even more suitable for complex flow configurations. Notably, it is well suited for any global dynamic procedure which adapts the overall model constant to the grid resolution and numerical errors.

\section{ACKNOWLEDGMENTS}

Hubert Baya Toda gratefully acknowledges support from IFP Energies Nouvelles. The authors acknowledge the MRI-R2 award for providing computing resources that have con- 
tributed to the research results reported within this paper. This award (Acquisition of a Hybrid CPU/GPU and Visualization Cluster for Multidisciplinary Studies in Transport Physics with Uncertainty Quantification) is funded under the American Recovery and Reinvestment Act of 2009 (Public Law 111-5). The authors also acknowledge CINES and IDRIS of GENCI for giving access to supercomputing facilities. Professors P. Moin and H. Choi are warmly acknowledged for fruitful discussions during the course of the 2010 Summer Program of the Center for Turbulence Research. Dr G. Lodato is thank for his comments on a preliminary version of this paper. At last, the authors are grateful to the anonymous reviewers for their constructive comments regarding the discussion of section III A.

\section{Appendix: Computation of the singular values of $\mathrm{g}$}

For each SGS viscosity assessment, the $\sigma$-model requires the computation of the three singular values of the local velocity gradient tensor. It is important that this computation is performed both efficiently and accurately. The two following methods were used with success during the course of this study:

- Method A: The first idea is to rely on optimized routines of linear algebra as available in scientific libraries as LAPACK. The computation of the $\sigma$ 's is then done in three steps:

1. build the matrix $\mathbf{G}=\mathbf{g}^{t} \mathbf{g}$ from the resolved velocity gradient. This $3 \times 3$ matrix is symmetric semi-definite positive and its eigenvalues are thus always positive,

2. compute the eigenvalues of $\mathbf{G}$, for example by using the SSYEV/DSYEV routines from LAPACK, and order them so that $\lambda_{1} \geq \lambda_{2} \geq \lambda_{3} \geq 0$,

3. compute the singular values of $\mathbf{g}$ from $\sigma_{1}=\sqrt{\lambda_{1}}, \sigma_{2}=\sqrt{\lambda_{2}}$ and $\sigma_{3}=\sqrt{\lambda_{3}}$.

- Method B: This method [44] is self-contained and does not require the use of an external scientific library. It consists in the following steps:

1. build the matrix $\mathbf{G}=\mathbf{g}^{t} \mathbf{g}$ from the resolved velocity gradient, 
2. compute its invariants as in Eq. (13), namely:

$$
\begin{aligned}
& \mathcal{I}_{1}=\operatorname{tr}(\mathbf{G}), \\
& \mathcal{I}_{2}=\frac{1}{2}\left(\operatorname{tr}(\mathbf{G})^{2}-\operatorname{tr}\left(\mathbf{G}^{2}\right)\right), \\
& \mathcal{I}_{3}=\operatorname{det}(\mathbf{G}),
\end{aligned}
$$

where the square of $\mathbf{G}$ is $\mathbf{G}_{i j}^{2}=\mathbf{G}_{i k} \mathbf{G}_{k j}$,

3. compute the following angles from the above invariants:

$$
\begin{aligned}
\alpha_{1} & =\frac{\mathcal{I}_{1}^{2}}{9}-\frac{\mathcal{I}_{2}}{3}, \\
\alpha_{2} & =\frac{\mathcal{I}_{1}^{3}}{27}-\frac{\mathcal{I}_{1} \mathcal{I}_{2}}{6}+\frac{\mathcal{I}_{3}}{2}, \\
\alpha_{3} & =\frac{1}{3} \arccos \frac{\alpha_{2}}{\alpha_{1}^{3 / 2}},
\end{aligned}
$$

4. compute the singular values as:

$$
\begin{aligned}
\sigma_{1} & =\left(\frac{\mathcal{I}_{1}}{3}+2 \sqrt{\alpha_{1}} \cos \alpha_{3}\right)^{1 / 2} \\
\sigma_{2} & =\left(\frac{\mathcal{I}_{1}}{3}-2 \sqrt{\alpha_{1}} \cos \left(\frac{\pi}{3}+\alpha_{3}\right)\right)^{1 / 2} \\
\sigma_{3} & =\left(\frac{\mathcal{I}_{1}}{3}-2 \sqrt{\alpha_{1}} \cos \left(\frac{\pi}{3}-\alpha_{3}\right)\right)^{1 / 2}
\end{aligned}
$$

From the experience gained during this study, the two above methods give virtually identical results. It is also interested to assess the computational cost related to these computations. Since the $\sigma$-model is proposed as an alternative to perform LES in complex geometries, Solver A (the general purpose solver, see Table III) was used for this purpose. Using Method A, the overall computation time required for a $64^{3}$ decaying turbulence case with the $\sigma$-model was approximately $10 \%$ larger than what is required with the static Smagorinsky model. Of course, this assessment may depend on the efficiency of the scientific library available on the target computer. Still, given the number of properties met by the $\sigma$ model compared to the Smagorinsky one, this extra cost is certainly worth being paid. More interestingly, the extra CPU cost becomes hardly measurable (less than $1 \%$ ) when Method $\mathrm{B}$ is used to compute the singular values. This method being moreover self-contained (no need for specific scientific libraries), it is certainly the best option to compute the required 
singular values.

[1] J. Smagorinsky. General circulation experiments with the primitive equations: 1 . the basic experiment. Mon. Weather Rev. , 91:99-164, 1963.

[2] P. Moin and J. Kim. Numerical investigation of turbulent channel flow. J. Fluid Mech. , 118:341-377, 1982.

[3] M. Germano, U. Piomelli, P. Moin, and W. Cabot. A dynamic subgrid-scale eddy viscosity model. Phys. Fluids, 3(7):1760-1765, 1991.

[4] E. Lévêque, F. Toschi, L. Shao, and J-P. Bertoglio. Shear-improved Smagorinsky model for large-eddy simulation of wall-bounded turbulent flows. J. Fluid Mech. , 570:491-502, 2007.

[5] F. Nicoud and F. Ducros. Subgrid-scale stress modelling based on the square of the velocity gradient. Flow, Turb. and Combustion, 62(3):183-200, 1999.

[6] A. Vreman. An eddy-viscosity subgrid-scale model for turbulent shear flow: Algebraic theory and applications. Phys. Fluids, 16(10):3670, 2004.

[7] F. Porté-Agel, C. Meneveau, and M. Parlange. A scale-dependent dynamic model for largeeddy simulation: application to a neutral atmospheric boundary layer. J. Fluid Mech. , 415:261-284, 2000 .

[8] T. J. R. Hughes, L. Mazzei, A. A. Oberai, and A. A. Wray. The multiscale formulation of large eddy simulation: Decay of homogeneous isotropic turbulence. Phys. Fluids , 13(2):505-512, 2001.

[9] H. Jeanmart and G. Winckelmans. Investigation of eddy-viscosity models modified using discrete filters: A simplified regularized variational multiscale model and an enhanced field model'. Phys. Fluids, 19(055110):1-12, 2007.

[10] L. Bricteux, M. Duponcheel, and G. Winckelmans. A multiscale subgrid model for both free vortex flows and wall-bounded flows. Phys. Fluids , 21:105102/1-12, 2009.

[11] Y. Shi, Z. Xiao, and S. Chen. Constrained subgrid-scale stress model for large eddy simulation. Phys. Fluids , 20(011701):1:4, 2008.

[12] G. Lodato, L. Vervisch, and P. Domingo. A compressible wall-adapting similarity mixed model for large-eddy simulation of the impinging round jet. Phys. Fluids, 21(3):035102, 2009. 
[13] J. Bardina, J. H. Ferziger, and W. C. Reynolds. Improved subgrid scale models for large-eddy simulations. Am. Inst. Aeronaut. Astronaut. J. , 34:1111-1119, 1980.

[14] Y. Zang, R. L. Street, and J. R. Koseff. A dynamic mixed subgrid-scale model and its application to turbulent recirculating flows. Phys. Fluids , 5(12):3186-3196, 1993.

[15] D. K. Lilly. A proposed modification of the germano sub-grid closure method. Phys. Fluids , 4(3):633-635, 1992.

[16] S. Ghosal, T. Lund, P. Moin, and K. Akselvoll. A dynamic localization model for large-eddy simulation of turbulent flows. J. Fluid Mech. , 286:229-255, 1995.

[17] C. Meneveau, T. Lund, and W. Cabot. A lagrangian dynamic subgrid-scale model of turbulence. J. Fluid Mech., 319:353-385, 1996.

[18] G. Ghorbaniasl and C. Lacor. Sensitivity of SGS models and of quality of LES to grid irregularity. In J. Meyers et al. (Eds), Quality and Reliability of Large-Eddy Simulations. Springer Science + Business Media B.V., 2008.

[19] H. Baya Toda, K. Truffin, and F. Nicoud. Is the dynamic procedure appropriate for all SGS models ? In J. C. F. Pereira and A. Sequeira, editors, V European Conference on Computational Fluid Dynamics, ECCOMAS, Lisbon, Portugal, 14-17 June 2010.

[20] C. B. Da Silva and O. Metais. On the influence of coherent structures upon interscale interaction in turbulent plane jets. J. Fluid Mech. , 473:103-145, 2002.

[21] N. Park, S. Lee, J. Lee, and H. Choi. A dynamic subgrid-scale eddy viscosity model with a global model coefficient. Phys. Fluids, 18:125109, 2006.

[22] D. You and P. Moin. A dynamic global-coefficient subgrid-scale eddy-viscosity model for large-eddy simulation in complex geometries. Phys. Fluids , 19:065110/1-8, 2007.

[23] J. Lee, H. Choi, and N. Park. Dynamic global model for large eddy simulation of transient flow. Phys. Fluids, 22:075106, 2010.

[24] D. Chapman and G. Kuhn. The limiting behavior of turbulence near a wall. J. Fluid Mech. , 170:265-292, 1986.

[25] M. Lesieur. Turbulence in Fluids. Springer Verlag - 4th edition, 2008.

[26] U. Frisch. Turbulence: The Legacy of A.N. Kolmogorov. Cambridge University Press, 1995.

[27] R. Verstappen. When does eddy viscosity restrict the dynamics to large eddies ? In J. C. F. Pereira and A. Sequeira, editors, V European Conference on Computational Fluid Dynamics, ECCOMAS, Lisbon, Portugal, 14-17 June 2010. 
[28] R. Verstappen, S. Bose, J. Lee H. Choi, and P. Moin. A dynamic eddy-viscosity model based on the invariants of the rate-of-strain. In Proc. of the Summer Program, pages 183-192. Center for Turbulence Research, NASA Ames/Stanford Univ., 2010.

[29] D. K. Lilly. The representation of small-scale turbulence in numerical simulation experiments. In Proceedings of the IBM Scientific Computing Symposium on Environmental Sciences, Yorktown Heights, USA, 1967.

[30] O. Colin and M. Rudgyard. Development of high-order taylor-galerkin schemes for unsteady calculations. J. Comput. Phys., 162(2):338-371, 2000.

[31] V. Moureau, G. Lartigue, Y. Sommerer, C. Angelberger, O. Colin, and T. Poinsot. Numerical methods for unsteady compressible multi-component reacting flows on fixed and moving grids. J. Comput. Phys., 202(2):710-736, 2005.

[32] O. Cabrit and F. Nicoud. Direct simulations for wall modeling of multicomponent reacting compressible turbulent flows. Phys. Fluids , 21(5):055108, May 2009.

[33] S. Mendez and F. Nicoud. Large-eddy simulation of a bi-periodic turbulent flow with effusion. J. Fluid Mech., 598:27-65, 2008.

[34] G. Comte-Bellot and S. Corrsin. Simple eulerian time correlation of full- and narrow-band velocity signals in grid generated, 'isotropic' turbulence. J. Fluid Mech. , 48:273-337, 1971.

[35] Y. Morinishi, T. Lund, O. Vasilyev, and P. Moin. Fully conservative higher order finite difference schemes for incompressible flow. J. Comput. Phys. , 143(1):90-124, 1998.

[36] H. S. Kang, S. Chester, and C. Meneveau. Decaying turbulence in an active-grid-generated flow and comparisons with large-eddy simulation. J. Fluid Mech. , 480, 2003.

[37] H. Baya Toda, O. Cabrit, G. Balarac, S. Bose, J. Lee, H. Choi, and F. Nicoud. A subgrid-scale model based on singular values for les in complex geometries. In Proc. of the Summer Program , pages 193-202. Center for Turbulence Research, NASA Ames/Stanford Univ., 2010.

[38] R. Cocle, L. Bricteux, and G. Winckelmans. Scale dependence and asymptotic very high reynolds number spectral behavior of multiscale subgrid models. Phys. Fluids, 21:085101/1$12,2009$.

[39] C. Meneveau and T. Lund. The dynamic smagorinsky model and scale-dependent coefficients in the viscous range of turbulence. Phys. Fluids , 9(12):3932-3934, 1997.

[40] J. Meyers and P. Sagaut. On the model coefficients for the standard and the variational multi-scale smagorinsky model. J. Fluid Mech. , 569:287319, 2006. 
[41] J. Meyers, C. Meneveau, and B. Geurts. Error-landscape-based multiobjective calibration of the smagorinsky eddy-viscosity using high-reynolds-number decaying turbulence data. Phys. Fluids , 22(12):125106, 2010.

[42] M. Lesieur and O. Metais. New trends in large-eddy simulations of turbulence. Ann. Rev. Fluid Mech. , 28:45 - 82, 1996.

[43] R.D. Moser, J Kim, and N.N. Mansour. Direct numerical simulation of turbulent channel flow up to $\operatorname{Re}_{\tau}=590 . \quad$ Phys. Fluids, 11(4):943-945, 1999.

[44] K. Hasan, P. Basser, D. Parker, and A. Alexander. Analytical computation of the eigenvalues and eigenvectors in dt-mri. J. of Magnetic Resonance, 152:41-47, 2001. 


\section{LIST OF FIGURES}

1 Time evolutions of the scaled kinetic energy for the freely decaying isotropic turbulence corresponding to the Comte-Bellot \& Corrsin [34] experiment. All computations performed with the general purpose solver AVBP (Solver A) and the $\sigma$-model with $C_{\sigma}=1.3(---) ; C_{\sigma}=1.4(\cdots \cdots \cdots) ; C_{\sigma}=1.5(-)$; $C_{\sigma}=1.6(-\cdot-) ; C_{\sigma}=1.7(--\cdot)$;. Symbols are experimental measurements corresponding to the three-dimensionless times $t U_{0} / M=42,98$ and 171. Top:

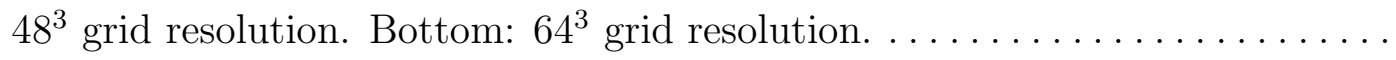

2 Time evolutions of energy spectra for the freely decaying isotropic turbulence corresponding to the Comte-Bellot \& Corrsin [34] experiment. Symbols are experimental measurements corresponding to the three-dimensionless times $t U_{0} / M=42,98$ and 171. Top: Results from the general purpose solver AVBP (Solver A) with grid resolution $64^{3}$ and $C_{\sigma}=1.5$. Bottom: Results from a spectral method (Solver C) with grid resolution $32^{3}$ and a global dynamic procedure applied to the $\sigma$-model.

3 Time evolution of model constant for the freely decaying isotropic turbulence corresponding to the Comte-Bellot \& Corrsin (CBC) [34] and Kang et al.[36] experiments. The computation is based on a spectral method (Solver C) and a global dynamic version of the $\sigma$-model. Grid resolution is $32^{3}$ and $128^{3}$ respectively.

4 Time evolutions of energy spectra for the freely decaying isotropic turbulence corresponding to the Kang et al.[36] experiment. Symbols are experimental measurements corresponding to the three-dimensionless times $t U_{0} / M=20$, 30, 40 and 48. Results are from the spectral Solver $\mathrm{C}$ with grid resolution $128^{3}$ and a global dynamic procedure applied to the $\sigma$-model. . . . . . . .

5 Mean velocity profile from the static $\sigma$-model ( - ) and the dynamic Smagorinsky model (--- ). Symbols correspond to the filtered DNS data [43]. Top: Results from the general purpose solver AVBP (Solver A) at $R_{\tau}=395$. Bottom: Results from the channel code (Solver B) at $R_{\tau}=590$. A no-model simulation $(. . . . . .$.$) is also shown in this latter case. ..........$ 
6 Velocity fluctuations (rms) from the static $\sigma$-model ( - ) and the dynamic Smagorinsky model (--- ). Symbols correspond to the filtered DNS data [43].

Top: Results from the general purpose solver AVBP (Solver A) at $R_{\tau}=395$.

Bottom: Results from the channel code (Solver B) at $R_{\tau}=590 \ldots \ldots \ldots \ldots 2$

7 Scaled SGS eddy-viscosity from the static $\sigma$-model ( - ) and the dynamic Smagorinsky model (--- ). The dotted lines correspond to the proper $y^{3}$ asymptotic behavior. Top: Results from general purpose solver AVBP (Solver

A) at $R_{\tau}=395$. Bottom: Results from the channel code (Solver B) at $R_{\tau}=590.26$ 


\section{LIST OF TABLES}

I Desirable properties for an improved SGS viscosity model. In the case of a static model, these properties should be met by the differential operator $\left(\mathcal{D}_{m}\right)$ the model is based on. . . . . . . . . . . . . . . . . . . .

II Properties of the SGS models considered. Labels Axisymmetric and Isotropic refer to axisymmetric and isotropic contraction/expansion respectively. The numerical entries in the $\mathbf{P} \mathbf{2}$ and $\mathbf{P} 3$ blocks are the values taken by the differential operators when all the velocity derivatives are zero except: Solid rotation: $d u_{1} / d x_{2}=-1$ and $d u_{2} / d x_{1}=1$; Pure shear: $d u_{1} / d x_{2}=1$; Axisymmetric: $d u_{1} / d x_{1}= \pm 2, d u_{2} / d x_{2}=\mp 1, d u_{3} / d x_{3}=\mp 1 ;$ Isotropic: $d u_{1} / d x_{1}= \pm 1$,

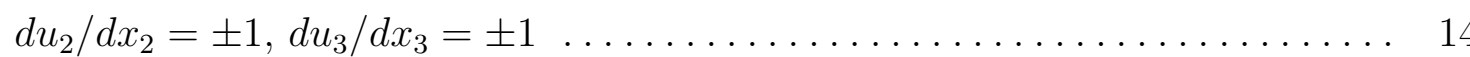

III Properties of the solvers A, B and C used to compute the academic cases considered. RK: Third-order Runge-Kutta. CN: Second-order Crank-Nicolson

IV Properties of the channel flow cases. $L_{x}, L_{y}$ and $L_{z}$ stand for the size of the computational domain in the streamwise, normalwise and spanwise directions and are scaled by the channel half-height $h ; N_{x}, N_{y}$ and $N_{z}$ are the corresponding number of grid cells. Grid spacings are given in classical wall units; the mesh is stretched in the $y$-direction to increase resolution in the near wall regions. $R_{\tau}$ stands for the friction Reynolds number based on the friction velocity and channel half-height. 